\title{
TERRITORIO DE EXPLOTACIÓN DE RECURSOS LÍTICOS EN LOS ANDES CENTRALES ARGENTINOS DURANTE EL PLEISTOCENO FINAL - HOLOCENO TEMPRANO
}

\author{
Alejandro García*
}

\begin{abstract}
GARCIA, A. Territorio de explotación de recursos líticos en los Andes Centrales Argentinos durante el Pleistoceno final - Holoceno temprano. Rev. do Museu de Arqueologia e Etnologia, São Paulo, 12: 17-24, 2002.
\end{abstract}

RESUMO: Os dados de artefatos líticos do abrigo Agua de la Cueva - Sector Sur, ao lado dos registros arqueológicos de fauna, dos recursos vegetais potencialmente disponíveis e das estruturas de recursos correntes da área, possibilitam o estudo de alguns aspectos relacionados à exploração do território do sítio durante o povoamento inicial dos Andes Centrais argentinos (ca. $11.000-7.500$ anos $C^{14}$ AP). São observadas uma acentuada tendência à captação de recursos locais e indicações de uma extensão trans-regional dos sistemas de assentamento/subsistência que incluíram o sítio durante o Pleistoceno final-Holoceno inicial.

UNITERMOS: Exploração de recursos - Andes Centrais argentinos - Transição Pleistoceno-Holoceno.

\section{Introducción}

Durante el Pleistoceno final el hombre ya explotaba variados ambientes de los Andes Centrales Argentino-Chilenos. La transición hacia condiciones climáticas más cálidas y el retroceso de los glaciares cordilleranos permitieron la ocupación de sectores montañosos hasta entonces inaccesibles. Uno de los sitios habitados en ese período se encuentra en el cordón precordillerano oriental de los Andes, a ca. 2.900 m s.n.m. Se trata de un extenso alero denominado Agua de la Cueva-Sector Sur, que funcionó como base residencial desde $c .11 .000$ años $\mathrm{C}^{14} \mathrm{AP}$ (García et al. 1999). Allí se recuperó un abundante registro arqueológico, constituido fundamentalmen-

(*) CONICET - UNSJ (FFHA), San Juan, Argentina. te por restos faunísticos y artefactos y desechos líticos. Este registro ofrece información relevante sobre variados aspectos relacionados con la movilidad y el aprovechamiento del espacio y los recursos. Esta clase de tópicos, provechosamente abordada en otras áreas (e.g. Vita Finzi et al. 1970; Bailey et al. 1983), ha sido escasamente tratada en la región debido a la escasez y características de la mayor parte del registro arqueológico pleistocénico, que corresponde a sitios interpretados como lugares de caza (Varela et al. 1993; Núñez et al. 1994; Lagiglia 1979). Para contribuir a llenar ese vacío, en el presente artículo se brinda información relacionada con las materias primas líticas y sus fuentes de aprovisionamiento, $\mathrm{y}$, tomando en consideración datos sobre los recursos faunísticos y vegetales, se analizan estos antecedentes en función de la determinación del alcance del territorio de explotación del sitio Agua de la Cueva - Sector Sur durante el Pleistoceno final y el Holoceno temprano. 
GARCIA, A. Territorio de explotación de recursos líticos en los Andes Centrales Argentinos durante el Pleistoceno final Holoceno temprano. Rev. do Museu de Arqueologia e Etnologia, São Paulo, 12: 17-24, 2002.

\section{El registro arqueológico y las fuentes de aprovisionamiento}

\section{Composición de la muestra analizada}

En el alero Agua de la Cueva - Sector Sur $\left(69^{\circ}\right.$ $09^{\circ} 49^{\prime \prime} \mathrm{W}, 32^{\circ} 37^{\circ} 01^{\prime \prime}$ S, 2.900 m s.n.m.) se registraron tres unidades estratigráficas (García et al. 1999) cuya duración se extiende desde el Pleistoceno final hasta el presente. Las primeras ocupaciones del sitio se localizaron en el inicio de la capa $2 \mathrm{~b}$ de la Unidad Estratigráfica 2 (Fig. 1) y fueron datadas en ca. 11.000 años $C^{14}$ AP (García 1998).

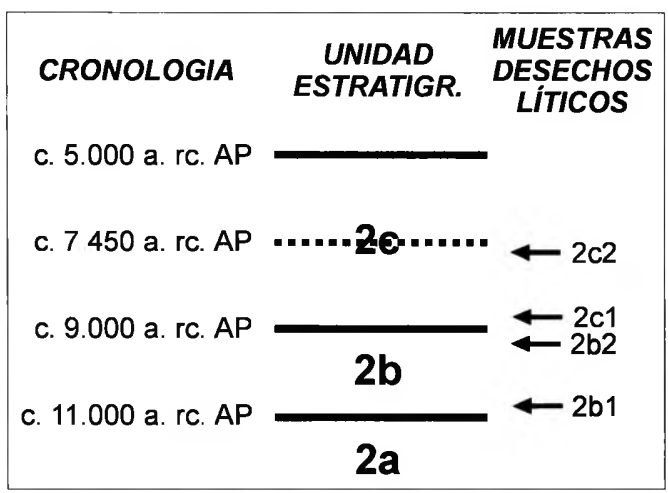

Fig. 1 - Inserción estratigráfica de las muestras de desechos líticos.

A fin de observar las tendencias generales en el uso de los recursos líticos en las ocupaciones de cada capa, la muestra analizada está constituida por el material de los niveles de excavación correspondientes al inicio y final de las capas $2 b$ (ca. 11.000 - ca. 9.000 años $\mathrm{C}^{14} \mathrm{AP}$ ), al inicio de $2 c$ (ca. 9.000 años $\mathrm{C}^{14} \mathrm{AP}$ ) y a un momento de 2c datado en $c a .7 .450$ años $\mathrm{C}^{14} \mathrm{AP}$ (Fig. 1). La muestra se obtuvo de la excavación de una superficie de $6 \mathrm{~m}^{2}$. Se analizaron 3.675 desechos líticos que representan el 20,4\% del total extraído. En cambio, debido a la gran variedad morfológica observada a priori, los instrumentos líticos $(n=828)$ fueron analizados en su totalidad. $\mathrm{El}$ carácter local, regional o exótico de las rocas depende de su ubicación hasta $5 \mathrm{~km}$, entre 5 y 20 km o a más de $20 \mathrm{~km}$ de distancia del sitio (Geneste 1988, cit. por Gamble 1993).

\section{La ubicación de los recursos líticos}

La variedad de las materias primas utilizadas para la talla de instrumentos durante las ocupaciones tempranas del sitio es amplia, pero existió una marcada preferencia por un grupo de rocas compuesto por la riolita, el cuarzo y la calcedonia (Tabla 1 y Fig. 2). El uso de otras rocas, como el esquisto, la toba, el basalto y la obsidiana, fue excepcional. También se registró una reducida cantidad de roca de grano fino no determinada que ha sido observada en el entorno del sitio. Proporciones similares entre las rocas locales y las no locales se observan en el conjunto de desechos (Tabla 1).

\section{TABLA 1}

\begin{tabular}{lcrrrrrr}
\hline \multirow{2}{*}{ Roca } & \multirow{2}{*}{ Proc. } & \multicolumn{2}{c}{ Total } & \multicolumn{1}{c}{ Capa 2b } & \multicolumn{2}{c}{ Capa 2c } \\
\cline { 3 - 9 } & & \multicolumn{1}{c}{ i } & \multicolumn{1}{c}{ d } & \multicolumn{1}{c}{ I } & \multicolumn{1}{c}{ d } & \multicolumn{1}{c}{ i } & d \\
\hline \hline Riolita & L & 468 & 884 & 425 & 625 & 43 & 259 \\
Cuarzo & L & 164 & 2261 & 137 & 632 & 27 & 629 \\
Calcedonia & L & 127 & 345 & 107 & 232 & 20 & 113 \\
Basalto & R & 14 & 14 & 13 & 14 & 1 & 0 \\
Cuarcita & R & 20 & 7 & 19 & 7 & 1 & 0 \\
Esquisto & L & 4 & 2 & 4 & 1 & 0 & 1 \\
Obsidiana & E & 1 & 1 & 1 & 1 & 0 & 0 \\
Otras locales & L & 7 & 0 & 7 & 0 & 0 & 0 \\
Indeterm. & L & 23 & 60 & 19 & 34 & 4 & 26 \\
\hline \multicolumn{1}{c}{ Total } & & 828 & 3674 & 732 & 2546 & 96 & 1128 \\
\hline
\end{tabular}

Tabla 1: Cantidad de instrumentos y desechos según materia prima. $\mathrm{L}=$ local, $\mathrm{R}=$ regional, $\mathrm{E}=$ exóticas

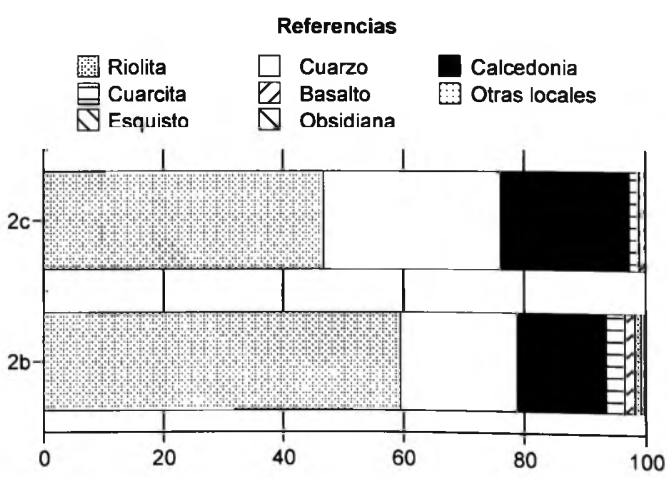

Fig. 2 - Porcentajes de instrumentos según materia prima.

Se han localizado diversas fuentes potenciales de aprovisionamiento de estas rocas (Fig. 3). Un cuarzo 
GARCIA, A. Territorio de explotación de recursos líticos en los Andes Centrales Argentinos durante el Pleistoceno final Holoceno temprano. Rev. do Museu de Arqueologia e Etnologia, São Paulo, 12: 17-24, 2002.

similar al del registro arqueológico fue ubicado en el sitio y en su entorno. Este cuarzo es duro y quebradizo (sensu Nami 1992), pero los afloramientos de la zona están surcados por abundantes planos internos de fractura. El cuarzo se presenta principalmente de dos maneras:

a) La forma más común es la de clastos de distintos tamaños producidos por la fragmentación de los afloramientos ubicados en torno al alero, que cubren las lomas situadas desde el sitio hasta una distancia de $c a .1 \mathrm{~km}$ por el oeste.

b) También aparecen grandes bloques de entre 1 y $5 \mathrm{~m}$ de largo en puntos cercanos al alero, aunque separados entre sí por distancias de hasta varios kilómetros. Por ejemplo, estos bloques han sido observados en Cerros Colorados, a ca. $2 \mathrm{~km}$ de
Agua de la Cueva, y en la quebrada que une la zona del Cerro Jagüelito con el alero (a ca.1 km de éste).

También se han descubierto varias fuentes potenciales de riolita. Esta materia es muy dura y en general poco quebradiza, y se presenta principalmente de tres formas:

a) Afloramientos de grandes bloques mayores de $1 \mathrm{~m}$ de largo, que aparecen en varios sectores del Cerro Jagüelito, donde la riolita es de color rojo o morado. También se ha hallado un bloque de riolita marrón, a una distancia de $c a .15 \mathrm{~km}$.

b) Clastos de diversos tamaños producidos por la fractura natural de aquellos afloramientos. Por ejemplo, esta situación se observa en la quebrada del Cerro Jagüelito y en algunas quebradas que descienden hacia el oeste (ca. $1 \mathrm{~km}$ de Agua de la Cueva); en estos casos la riolita es roja o morada.

c) Concentraciones de rodados, que han sido observadas en un conglomerado en los Cerros Colorados (ca. $2 \mathrm{~km}$ hacia el WNW) y en las laderas NE y E del Cerro Jagüelito (entre 2 y 4 $\mathrm{km}$ del sitio). En estos casos predomina el color marrón y, a diferencia de las riolitas rojas y moradas, la inclusión de fenocristales es abundante.

La calcedonia, aparece fundamentalmente de dos maneras:

a) Como rodados de tamaño generalmente pequeño, tanto en el conglomerado ubicado en Cerros Colorados como en varias concentraciones localizadas entre 2 y $4 \mathrm{~km}$ hacia el NE y E. Los colores más frecuentes en estos casos son los rojizos, marrones y verdosos.

b) Como fragmentos en forma de "rodajas" producidos por la meteorización que actuó seccionando transversalmente rodados de tamaño mediano en el sitio Cerros Colorados.
Fig. 3 - Probables fuentes de aprovisionamiento de materias primas líticas. 1. Cerros Colorados (calcedonias y riolitas); 2. Afloramientos de cuarzo en torno al sitio; 3. Rodados de calcedonia; 4. Riolitas y calcedonias; 5-6. Riolitas rojas y moradas (Quebr. del Jagüelito); 7-8-9. Afloramientos de riolita; 10. Rodados de riolita marrón con fenocristales; 11 . Rodados de calcedonia. 
GARCIA, A. Territorio de explotación de recursos líticos en los Andes Centrales Argentinos durante el Pleistoceno final Holoceno temprano. Rev. do Museu de Arqueologia e Etnologia, São Paulo, 12: 17-24, 2002.

Sólo se han observado afloramientos pequeños (menos de $1 \mathrm{~m}$ de extensión) en la cumbre del Cerro Jagüelito; en este caso se trata de una calcedonia de color beige con numerosos planos de fractura.

En los pocos casos de utilización de esquisto (roca sumamente degradable) se trata de fragmentos desprendidos del techo y paredes del alero.

Con respecto a la cuarcita, los estudios geológicos locales indican la presencia de guijarros en una tillita paleozoica distante unos $10 \mathrm{~km}$ del sitio (Keidel 1938:31-32; García 1951). Guijarros similares han sido observados en las inmediaciones del sitio Cueva del Toro, a ca. $15 \mathrm{~km}$ hacia el sur.

Finalmente, el basalto sólo ha sido hallado en algunos sectores aledaños al río Mendoza (15$20 \mathrm{~km}$ hacia el sur), en tanto que las fuentes de obsidiana más próximas se ubican a más de 100 $\mathrm{km}$ al sur del sitio.

\section{Variaciones en el registro e implicancias}

Aun teniendo en cuenta el tamaño disímil de la muestra de cada capa, la comparación entre $2 b$ y $2 \mathrm{c}$ muestra algunas diferencias cuantitativas importantes, que permiten plantear alternativas vinculadas con la explotación de los recursos precordilleranos y con la movilidad de los grupos humanos tempranos. En este sentido, resaltan dos aspectos: el decrecimiento del arribo de rocas no locales y, en contrapartida, el aumento relativo de la explotación de un recurso cercano específico: la riolita roja/morada.

1) La primera tendencia está evidenciada por la marcada diferencia de instrumentos y desechos de talla elaborados en rocas no locales entre las capas $2 b$ y $2 c$ (Tabla 2 ).

\section{TABLA 2}

\begin{tabular}{ccccccccc}
\hline & \multicolumn{3}{c}{ Obsidiana } & \multicolumn{2}{c}{ Cuarcita } & \multicolumn{2}{c}{ Basalto } & \multicolumn{2}{c}{ Total } \\
& i & d & i & d & i & d & i & d \\
\hline \hline $2 \mathrm{~b}$ & 1 & 1 & 19 & 7 & 13 & 14 & 33 & 22 \\
$2 \mathrm{c}$ & 0 & 0 & 1 & 0 & 1 & 0 & 2 & 0 \\
\hline
\end{tabular}

Tabla 2: Cantidad de instrumentos ("i") y desechos de talla ("d") en rocas no locales.
De los 22 desechos líticos de 2b, 17 (77\%) corresponden a la muestra 2b1 (Fig. 1), lo que implica una diferencia significativa de la presencia de desechos de materias primas no locales entre las primeras ocupaciones del sitio y las correspondientes al Holoceno temprano.

En relación a la talla de instrumentos, en $2 c$ se destaca la mínima representación de basalto y cuarcita $(2 \%)$ y la ausencia de obsidiana, en tanto se acentúa el uso casi exclusivo de riolita, cuarzos y calcedonia (94\%) y la mayor utilización de la riolita de variedad roja/morada.

Las diferencias observables en las cantidades de desechos e instrumentos elaborados en rocas no locales indican una reducción importante de su ingreso al sitio desde $c a$. 9.000 años $\mathrm{C}^{14} \mathrm{AP}$, lo que podría vincularse con un menor conocimiento de los recursos líticos de las cercanías del alero durante las ocupaciones pleistocénicas. El cambio mencionado podría reflejar fundamentalmente dos situaciones:

a) Una restricción del acceso u ocupación de algunas zonas (e.g. el extremo meridional precordillerano y el sector ubicado a más de $c a .10$ $\mathrm{km}$ al oeste del sitio) hacia 9.000 años $\mathrm{C}^{14} \mathrm{AP}$. En este caso podría suponerse el abandono de la explotación de estas zonas a raíz de una variación del sistema de asentamiento, o de la imposibilidad de acceso por problemas de territorialidad.

b) Un cambio en la organización de la tecnología (Nelson 1991) y en las conductas de explotación de los recursos líticos en torno al sitio (vinculado probablemente con un mayor conocimiento del territorio), caracterizado por la intensificación de la explotación de los recursos locales; el acceso a los demás sectores de la precordillera pudo mantenerse abierto, en tanto que la movilización de sus recursos líticos se restringía a unos pocos instrumentos conservados.

2) La otra tendencia importante está marcada por el aumento de los desechos de riolita roja/ morada en relación al total de riolita y al total de desechos (Fig. 4). En la muestra de desechos de ca. 11.000 años $\mathrm{C}^{14} \mathrm{AP}$ la variedad roja representaba el $24 \%(n=82)$ del total de riolitas $(n=342)$, en tanto que el porcentaje se elevó sucesivamente en las muestras correspondientes a ca. $9.000 \mathrm{y} \mathrm{a} c a$. 7.500 años $\mathrm{C}^{14} \mathrm{AP}$ a 40,55 y $81 \%$ respectiva- 
GARCIA, A. Territorio de explotación de recursos líticos en los Andes Centrales Argentinos durante el Pleistoceno final Holoceno temprano. Rev. do Museu de Arqueologia e Etnologia, São Paulo, 12: 17-24, 2002.

mente. Algo similar se observa al comparar las riolitas rojas con el total de rocas entre $c a .11 .000$ y ca. 7.450 años $\mathrm{C}^{14} \mathrm{AP}$, ya que se pasó de 8 a $34 \%$.

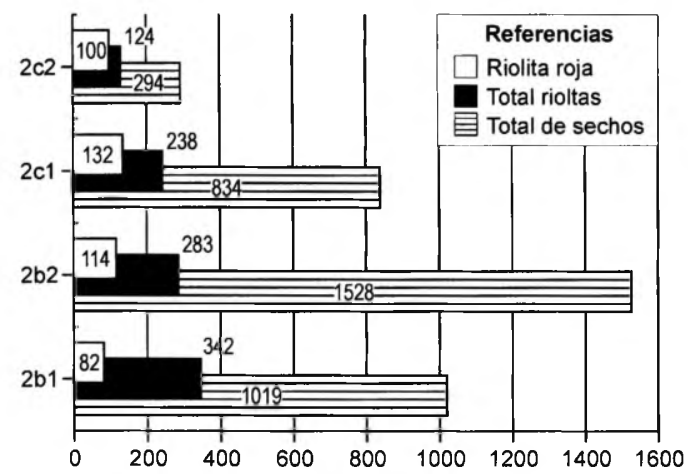

Fig. 4 - Relación entre cantidades de desechos de talla, total de riolitas y riolitas rojas.

Dado que la localización de la variedad roja/morada es bastante restringida (Cerro Jagüelito y alrededores), los datos obtenidos indicarían un mayor aprovechamiento de los recursos de esa zona durante el Holoceno temprano. Si bien esta conducta podría vincularse con una mayor explotación de los recursos faunísticos de la Pampa de Canota o con la simple preferencia por la riolita roja sobre las demás variedades, el hecho de que los instrumentos de riolita roja no se manufacturaran en la zona de aprovisionamiento (donde además se localiza una pequeña cueva habitable) sugiere un aprovisionamiento ocasional que podría estar relacionado con la vía de arribo de los grupos humanos a Agua de la Cueva.

Por último, la presencia de obsidiana es muy significativa, ya que su obtención podría indicar la extensión del sistema de asentamiento de los primeros ocupantes del alero, o la existencia de redes de intercambio que incluían el centro y norte de Mendoza ya en el Pleistoceno final. Asimismo, la presencia de basalto sugiere una vinculación con los territorios del extremo meridional precordillerano y del piedemonte oriental (donde se ubica el sitio La Crucesita, que ofrece gran cantidad de basalto y donde se realizó el hallazgo de una punta "cola de pescado" elaborada en ese material (Schobinger 1971).

\section{La explotación de recursos faunísticos y vegetales}

Los análisis arqueofaunísticos (Neme et al. 1998) indican el marcado predominio de restos de Artiodactyla, y la muy escasa presencia de elementos de Dasypodidae, Lagidium, Rheidae y Rodentia. La mayoría de los restos de Artiodactyla corresponden a Lama sp. y Lama guanicoe; en la capa 2b también aparecen escasos restos del sub-género Lama (Vicugna) - sensu Menegaz et al. 1989 -, que a nivel de especie podrían corresponder a la extinta gracilis o a la actual vicuña. Excepto Lama (Vicugna), el registro faunístico coincide con la oferta actual de los alrededores del sitio.

Según el registro analizado el principal recurso de origen animal fue el guanaco. En la actualidad, los lugares más atractivos de la zona para las tropillas de guanaco son la Pampa de Canota (Fig. 3), que ofrece una extensa cubierta de Stipa sp., y las quebradas abrigadas al norte, este y oeste de aquélla, que brindan aguadas y vegas de diversa extensión. Además, la vertiente permanente de Agua de la Cueva debió jugar un papel muy importante en épocas secas, como agente concentrador de las poblaciones animales. También existen otras aguadas menores que pudieron funcionar simultáneamente, posibilitando el alejamiento de las tropillas con respecto al sitio y, en consecuencia, disminuyendo la capacidad de sustento de su entorno inmediato. Tal situación, por tanto, pudo elevar eventualmente el costo energético de la caza mayor. No obstante, las posibilidades de hallar tropillas de guanacos en un radio de 3 a $5 \mathrm{~km}$ del sitio debieron ser constantemente altas. Las condiciones paleoambientales hacia el Pleistoceno final-Holoceno temprano (Páez 1997; Zárate et al. 1998) en la precordillera mendocina sugieren el mayor desarrollo de una estepa arbustiva de tipo patagónico, que habría posibilitado una densidad de camélidos similar o mayor a la actual que a su vez pudo convertir a este recurso en abundante y altamente predecible. Coincidentemente, la ausencia de selectividad de partes esqueletarias (Neme et al. 1996) sugiere que las cacerías se realizaban a una distancia relativamente corta del alero.

La obtención de recursos menores como Lagidium viscacia, que actualmente habita en los roquedales situados entre 1 y $3 \mathrm{~km}$ al sureste (Cerro 
Jagüelito), y la recolección de huevos de ñandú, cuyas cáscaras aparecen a lo largo de toda la secuencia temprana, constituyeron actividades complementarias $o$ alternativas que pudieron desarrollarse en las inmediaciones de Agua de la Cueva.

Por su parte, la oferta de recursos vegetales (al igual que en los tiempos modernos) debió ser muy restringida. Las condiciones más frías y probablemente más húmedas anteriores a $c a .9 .000$ años C ${ }^{14}$ AP (Páez 1997; García et al. 1999) se relacionan fundamentalmente con una vegetación de pastizales y con una reducida representación de taxa de monte, lo que implica una menor oferta de especies arbustivas, aunque suficiente para hacer frente a la demanda de leña (fundamentalmente Schinus $s p$. y Adesmia $s p$.). En rigor, las únicas evidencias de macrorrestos vegetales son precisamente los carbones hallados en los fogones. Por otro lado, aun suponiendo una cubierta vegetal similar a la actual, en las cercanías del sitio sólo se habrían podido recolectar cactáceas, cuyo consumo se evidencia en otros sitios de la región en épocas posteriores (García 1988; Bárcena et al. 1982).

\section{Conclusiones}

La comparación entre el registro arqueológico del sitio y la actual estructura de recursos similares a los utilizados por los grupos cazadoresrecolectores tempranos de Agua de la Cueva sugiere una extensión restringida del área de captación de materias primas líticas y de algunos alimentos de origen animal. En relación al aprovisionamiento de rocas para la talla de instrumentos, la movilidad de los ocupantes del sitio durante la transición Pleistoceno-Holoceno pudo mantenerse estrechamente limitada a un radio no mayor de 4 ó $5 \mathrm{~km}$; esta conducta no muestra alteraciones significativas a lo largo de las ocupaciones de las capas $2 b$ y $2 c$. En el mismo sentido, no se han hallado evidencias de un ingreso importante de recursos alóctonos, aunque en el caso de los vegetales (eventuales indicadores de una probable explotación logística de ambientes ubicados a más de $20 \mathrm{~km}$ del sitio) su ausencia podría deberse a problemas de conservación del registro. Este patrón de explotación de recursos a nivel fundamentalmente local coincide con la tendencia observada en otros sitios tempranos sudamericanos (Borrero 1996:348).

La presencia de rocas regionales (basalto, cuarcita) y alóctonas (obsidiana) no ofrece información sobre la movilidad logística de los grupos que habitaban el alero sino sobre la probable ubicación de otros componentes de los diversos sistemas de asentamiento-subsistencia que incluyeron al sitio a lo largo del Pleistoceno final-Holoceno temprano. La obtención de algunos recursos en los límites de la región montañosa precordillerana (basalto) y muy lejos de ésta (obsidiana) sugiere que algunos de estos sistemas tenían un alcance transregional que habría involucrado ambientes adyacentes como el piedemonte y la llanura oriental mendocina.

\section{Agradecimientos}

Agradezco a Cristina Bayón sus valiosos comentarios y sugerencias al manuscrito. El estudio del sitio Agua de la Cueva ha contado con el apoyo económico y logístico de la Fundación Antorchas, de la Secretaría de Ciencia y Técnica de la UNCuyo, de la ANPCyT y de la Facultad de Filosofía y Letras (UNCuyo) 
GARCIA, A. Territorio de explotación de recursos líticos en los Andes Centrales Argentinos durante el Pleistoceno final Holoceno temprano. Rev. do Museu de Arqueologia e Etnologia, São Paulo, 12: 17-24, 2002.

GARCIA, A. Lithic resources exploitation territory in the Argentinean Central Andes during the late Pleistocene - early Holocene. Rev. do Museu de Arqueologia e Etnologia, São Paulo, 12: 17-24, 2002.

ABSTRACT: Data on lithic artifacts from Agua de la Cueva - Sector Sur shelter, along with faunal archaeological records, potentially available vegetable resources and current structure of resources of the area, allow for the study of some aspects related to the site exploitation territory during the early peopling ( $c a .11,000-7,500 \mathrm{C}^{14}$ years BP) of the Andes Centrales Argentinos. A marked tendency to the catchment of local resources and indications of a transregional extension of the settlement/subsistence systems that included the site during the late Pleistocene - early Holocene are observed.

UNITERMS: Resouces exploitation - Argentinean Central Andes - PleistoceneHolocene transition.

\section{Referencias bibliográfícas}

\section{BAILEY, G.; DAVIDSON, T.}

1983 Site exploitation territories and topography. Two cases studies from Paleolithic Spain. Journal of Archaeological Science, 10: 87-115.

BÁRCENA, J.R.; ROIG, F.A.

1981-2 Investigaciones arqueológicas en el área puneña de Mendoza, con especial referencia a Tephrocactus andícola (Cactaceae) como nuevo recurso alimentario. Relaciones de la Sociedad Argentina de Antropología, XIV (2): 85-107.

BORRERO,L.

1996 The Pleistocene-Holocene Transition in Southern South America. L.G. Straus, B.V. Eriksen, J.M. Erlandson; D.R. Yesner (Eds.) Humans at the End of the Ice Age; the archaeology of the Pleistocene-Holocene Transition. New York, Plenum: 339-354.

GAMBLE, C.

1993 Exchange, Foraging and Local Hominid Networks. C. Scarre; F. Healy (Eds.) Trade and Exchange in Prehistoric Europe. Oxford. Oxbow Books: 35-54.

GARCIA, E.

1951 Contribución al conocimiento de la precordillera mendocina. Actas de la XV Semana de Geografía. Universidad Nacional de Cuyo; San Juan, Mendoza y San Luis: 491-507.

GARCÍA, A.

1988 Arqueología de la Cueva del Toro (Mendoza, Argentina). Revista de Estudios Regionales 1: 17-71.

1998 Geocronología del alero Agua de la Cueva Sector Sur. Revista de Estudios Regionales, 17: 49-64.
GARCÍA, A.; ZÁRATE, M.; PÁEZ, M.

1999 The Pleistocene-Holocene Transition and Human Occupation in the Central Andes of $\mathrm{Ar}$ gentina: Agua de la Cueva locality. Quaternary International, 53/54: 43-52.

GENESTE, J.M.

1988 Systemes d'approvisionnement en matières premières au Paleolithique Moyen et au Paleolithique Superieur en Aquitaine. L'Homme de Néandertal, 8: 61-70.

KEIDEL, J.

1938 Las estructuras de corrimientos paleozoicos de la sierra de Uspallata (Prov. de Mendoza). Physis, XIV (46): 1-96.

LAGIGLIA, $\mathrm{H}$.

1979 Dinámica cultural en el centro oeste y sus relaciones con áreas aledañas argentinas y chilenas. Actas del VII Congreso de Arqueología de Chile, II, Altos de Vilches: 531-560.

MENEGAZ, A.; GOIN, F.; ORTIZ JAUREGUIZAR, E.

1989 Análisis Morfométrico Multivariado de los Representantes Fósiles y Vivientes del Género Lama (Artiodactyla, Camelidae). Sus Implicancias Sistemáticas, Biogeográficas, Ecológicas y Biocronológicas. Ameghiniana. 26: $153-172$.

NAMI, G.

1992 El subsistema tecnológico de la confección de instrumentos líticos y la explotación de los recursos del ambiente: una nueva vía de aproximación. Shincal, 2: 33-53.

NELSON, M.

1991 The Study of Technological Organization. M Schiffer (Ed.) Archaeological Method and 
GARCIA, A. Territorio de explotación de recursos líticos en los Andes Centrales Argentinos durante el Pleistoceno final Holoceno temprano. Rev. do Museu de Arqueologia e Etnologia, São Paulo, 12: 17-24, 2002.

Theory, vol. 3. Tucson, The University of Arizona Press: $57-100$.

NEME, G.; GIL, A.

1996 Arqueofauna Pleistoceno Final/Holoceno Temprano de Agua de la Cueva (Mendoza). $\mathrm{Ms}$

NEME, G.; GIL, A.; GARCÍA, A.

1998 Preliminary Zooarchaeological Results at Agua de la Cueva Sector Sur Shelter. Current Research in the Pleistocene, 15: 139-140.

NÚÑEZ, L.; VARELA, J.; CASAMIQUELA, R.; SCHIAPPACASSE, V.; NIEMEYER F.H.; VILLAGRÁN; C.

1994 Cuenca de Taguatagua en Chile: el ambiente del Pleistoceno y ocupaciones humanas. Revista Chilena de Historia Natural, 67 (4): 503-519.

NÚÑEZ,L;:VARELA,J.;CASAMIQUELA, R.; VILLAGRÁN,C.

1994 Reconstrucción multidisciplinaria de la ocupación prehistórica de Quereo, centro de Chile. Latin American Antiquity, 5 (2): 99-118.

PÁEZ, M

1997 Reconstrucción de la historia de la vegetación en la precordillera de Mendoza entre los 12.000 - 5000 años AP: localidad arqueológica de Agua de la
Cueva. Resúmenes del X Simposio Argentino de Paleobotánica y Palinología, Mendoza: 36.

SCHOBINGER, J.

1971 Una punta de tipo "cola de pescado" de la Crucesita (Mendoza). Anales de Arqueología y Etnología, XXVI: 89-97.

VARELA, J; NÚÑEZ, L.; CASAMIQUELA, $R$.

1993 Geología del Cuaternario de la Depresión Central de Chile entre Santiago y Laguna de Taguatagua. Taller Internacional "El Cuaternario de Chile". Santiago.

VITA FINZI, C.; HIGGS, E.

1970 Prehistoric Economies in the Mount Carmel Area: Site Catchment Analysis. Proceedings of the Prehistoric Society, 36: 1-37.

ZÁRATE ,M.; PÁEZ, M.; GARCÍA, A.

1998 Condiciones ambientales entre los 14000 y los 5000 años $\mathrm{C}^{i 4}$ en la Precordillera de Mendoza, Argentina: Localidad Agua de la Cueva. Resumos expandidos. VI Congresso da Associação Brasileira de Estudos do Quaternário (ABEQUA) e Reunião sobre o Quaternário da América do Sul. Curitiba: 517-521.

Recebido para publicação em 7 de novembro de 2002. 